\title{
Carbon monoxide releasing molecule-2 (CORM-2) inhibits growth of multidrug- resistant uropathogenic Escherichia coli in biofilm and following host cell colonization
}

Charlotte Sahlberg Bang ${ }^{1,2}$, Robert Kruse ${ }^{1,2}$, Kjell Johansson ${ }^{1}$ and Katarina Persson ${ }^{1,2^{*}}$

\begin{abstract}
Background: Increased resistance to antimicrobial agents is a characteristic of many bacteria growing in biofilms on for example indwelling urinary catheters or in intracellular bacterial reservoirs. Biofilm-related infections caused by multidrug-resistant bacteria, such as extended-spectrum $\beta$-lactamase (ESBL)-producing Enterobacteriaceae, are a major challenge. The aim of this study was to investigate if a carbon monoxide-releasing molecule (CORM-2) has antibacterial effects against ESBL-producing uropathogenic E. coli (UPEC) in the biofilm mode of growth and following colonization of host bladder epithelial cells.
\end{abstract}

Results: The effect of CORM-2 was examined on bacteria grown within an established biofilm (biofilm formed for $24 \mathrm{~h}$ on plastic surface) by a live/dead viability staining assay. CORM-2 (500 $\mu \mathrm{M}$ ) exposure for $24 \mathrm{~h}$ killed approximately $60 \%$ of the ESBL-producing UPEC isolate. A non-ESBL-producing UPEC isolate and the E. coli K-12 strain TG1 were also sensitive to CORM-2 exposure when grown in biofilms. The antibacterial effect of CORM-2 on planktonic bacteria was reduced and delayed in the stationary growth phase compared to the exponential growth phase. In human bladder epithelial cell colonization experiments, CORM-2 exposure for $4 \mathrm{~h}$ significantly reduced the bacterial counts of an ESBL-producing UPEC isolate.

Conclusion: This study shows that CORM-2 has antibacterial properties against multidrug-resistant UPEC under biofilmlike conditions and following host cell colonization, which motivate further studies of its therapeutic potential.

Keywords: Carbon monoxide releasing molecule, CORM-2, Extended-spectrum $\beta$-lactamase, Uropathogenic Escherichia coli, Biofilm

\section{Background}

Biofilm is defined as bacteria enclosed in a self-produced polymeric matrix adherent to a biotic or abiotic surface [1]. Escherichia coli (E. coli) growing in biofilm display a development in several steps. Attachment is first mediated by flagella, type 1 fimbriae, curli and polysaccharides followed by early development of biofilm architecture and a maturation step [2]. Bacterial biofilms are more resistant to the effects of an antimicrobial agent than planktonic

\footnotetext{
*Correspondence: katarina.persson@oru.se

${ }^{1}$ Faculty of Medicine and Health, Örebro University, SE-701 82 Örebro, Sweden

${ }^{2}$ RiSC - Inflammatory Responses and Infection Susceptibility Centre, Örebro University, SE-701 82 Örebro, Sweden
}

bacteria [3]. Slow growth rates, induction of biofilmspecific phenotypes and stress response activation as well as restricted antibiotic penetration by biofilm architecture have been proposed to explain the reduced susceptibility [3]. Biofilm formation on indwelling devices such as urinary catheters increases the risk of urinary tract infections (UTIs) and results in considerable antibiotic use [4]. Uropathogenic isolates of E. coli (UPEC) are the most frequent isolate in catheterized patients with UTI symptoms [5], and biofilm producing UPEC are also more frequent among strains causing UTI relapse [6]. Moreover, it has recently been recognized that UPEC may form biofilmlike structures on and inside bladder epithelial cells [7, 8]. If UPEC invade the urothelial cells they may rapidly 
replicate and subsequently aggregate into biofilm-like intracellular bacterial communities (IBC). In the late stage of IBC formation, the IBCs break open causing flux of bacteria from the superficial bladder facet cells into the bladder lumen allowing for invasion of other bladder cells $[7,9]$. UPEC have also been shown to establish small clusters of more persistent intracellular reservoirs within the underlying basal epithelium [10]. Intracellular reservoirs of UPEC are believed to go undetected by standard urine culture, be protected from host defense mechanisms and persist despite antibiotic therapy [9]. Interestingly, emerging data suggest that UPEC persisting within intracellular reservoirs may have a role in development of recurrent and chronic UTIs $[8,9,11]$. Commonly used antibiotic treatments failed to eradicate UPEC growing internalized within bladder epithelial cells, by a proposed combination of biofilm formation, metabolically quiescent bacteria and bladder urothelial barriers [12, 13]. Thus, treatment of biofilm-related UTI is a challenge and particularly if caused by multidrug-resistant UPEC isolates.

Multidrug-resistant extended-spectrum $\beta$-lactamase (ESBL)-producing E. coli have disseminated worldwide and a main reason for mortality caused by these bacteria is inadequate initial antimicrobial therapy [14]. ESBLproducing Enterobacteriaceae spp. have genes that code for the ESBL enzyme and different ESBL enzyme variants (TEM, SHV, CTX-M) have been identified. CTX-M $\beta$-lactamases confer resistance to third generation cephalosporins, like cefotaxime, and often against other classes of antibiotics such as fluoroquinolones [15]. Dissemination of ESBL-producing bacteria occurs by horizontal transfer of plasmids (mainly CTX-M plasmids) or clonal spread [16]. The CTX-M-15 dominance around the world is due to dissemination of the virulent UPEC clone ST131 [17]. Studies indicate that the close contact between cells during biofilm conditions facilitates plasmid transfer by conjugation, a phenomenon that may increase the development of multidrug resistance in biofilm [18].

New treatment strategies for multidrug-resistant bacteria may include approaches with more penetrant antimicrobials with activity against non-growing bacteria or biofilm. Carbon monoxide (CO) is a small gaseous molecule with anti-inflammatory and antimicrobial properties that is able to penetrate cell membranes [19]. Metal carbonyl compounds, commonly known as CO-releasing molecules or CORMs, that release $\mathrm{CO}$ in a controlled manner have been developed for therapeutic applications [20]. Antibacterial effects of ruthenium-based carbonyls (CORM-2 and CORM-3) are reported in E. coli K-12 strains, Staphylococcus aureus and Pseudomonas aeruginosa [21-23]. CORM-3 has been shown to rapidly deliver $\mathrm{CO}$ to the intracellular part of the bacteria and to inhibit respiration by reacting with cytochrome $b d$ and $b o^{\prime}$ [22]. However, the activity of CORMs is not restricted to impairment of the aerobic respiratory chain, as these compounds are also effective in near-anaerobic conditions [21]. Another proposed mechanism for the antibacterial effect of CORM-2 is generation of intracellular reactive oxygen species that cause DNA damage and death [24]. Many genes related to biofilm formation were modified by CORM- 2 as shown by whole-genome transcription profiling of a non-pathogenic E. coli $\mathrm{K}-12$ strain [25]. We have recently shown that CORM-2 has bactericidal effects on planktonic multidrug-resistant ESBL-producing UPEC isolates [26], but it is not yet known if CORM-2 will be effective against ESBLproducing UPEC isolates in biofilms.

The aim of the present work was to investigate the antibacterial effects of CORM-2 on ESBL-producing UPEC in the biofilm mode of growth and following colonization of host bladder epithelial cells.

\section{Methods \\ Bacterial isolates and strains}

Four ESBL-producing UPEC isolates (designated ESBL1, $6,7,9)$ and four non-ESBL-producing UPEC isolates (designated UPEC 2, 3, 4, 5) were obtained from the Department of Microbiology at Örebro University hospital, Sweden. The UPEC isolates were recovered from urine of standard care patients with indwelling urinary catheters and symptoms of UTI. The identity of the patients was anonymized prior to further analysis of the bacterial isolates. Ethical approval was not required for this study as no analysis of human subjects, human material or human data were made. Antimicrobial susceptibility testing was performed using methods recommended by the Swedish Reference Group for Antibiotics (www.sls.se/ RAF) and the subcommittee on methodology (NordicAST) (www.nordicast.org). The ESBL-producing isolates were characterized regarding CTX-M type as previously described [27]. The E. coli clone ST131 was detected using two ST131-specific pabB SNP assays by real-time PCR as previously described [28]. The CTX-M types, clonal group ST131 and the antibiotic susceptibility of the ESBL-producing isolates are shown in Table 1. The commensal E. coli $\mathrm{K}-12$ strain TG1 carrying a Fconjugative plasmid that promotes biofilm formation [29] was used as a positive control strain for biofilm formation. TG1 and the commensal E. coli $\mathrm{K}-12$ strain MG1655 were used from laboratory stocks.

\section{Analysis of biofilm formation}

Overnight cultures in Difco Luria-Bertani (LB) broth (Lennox, Becton Dickinson, Franklin Lakes, USA) were used to inoculate (at $0.1 \%$ ) fresh minimal salt (MS) medium [21] on agitation to an optical density $\left(\mathrm{OD}_{620}\right)$ of approximately 0.05 . The bacteria were seeded into 96- 
Table 1 Characteristics of the clinical isolates

\begin{tabular}{|c|c|c|c|c|}
\hline Isolate & CTX-M subgroup & CTX-M type & ST131 clone & Antibiotic resistance \\
\hline ESBL 1 & CTX-M -9 & CTX-M-24 & - & CTX, CAZ, CTB, CIP, GEN, MEL, TMP \\
\hline ESBL 6 & CTX-M -1 & CTX-M-15 & + & CTX, CAZ, CTB, CIP, TMP \\
\hline ESBL 7 & CTX-M -1 & CTX-M-15 & + & CTX, CAZ, CIP, MEL, TMP \\
\hline ESBL 9 & CTX-M -9 & CTX-M-14 & - & CTX, CAZ, CIP, MEL, TMP \\
\hline \multicolumn{5}{|l|}{ UPEC $2^{a}$} \\
\hline \multicolumn{5}{|l|}{ UPEC $3^{\mathrm{a}}$} \\
\hline UPEC $4^{a}$ & & & & TMP \\
\hline UPEC $5^{\mathrm{a}}$ & & & & CIP \\
\hline
\end{tabular}

Antibiotic resistance of ESBL-producing isolates (ESBL) and non-ESBL-producing UPEC isolates (UPEC) isolated from urine of patients with UTI. The CTX-M subgroup, CTX-M type and sequence type (ST) 131 are indicated for the ESBL-producing isolates

Abbreviations: cefotaxime (CTX), ceftazidime (CAZ), ceftibuten (CTB), ciprofloxacin (CIP), gentamicin (GEN), mecillinam (MEL), nitrofurantoin (NIT), trimethoprim (TMP)

${ }^{\mathrm{a}}$ susceptibility tested for NIT, MEL, TMP, CTX, CIP

well plastic plates (Nunc C96 Microwell plate, Nunc A/ $\mathrm{S}$, Roskilde, Denmark) and after $6 \mathrm{~h}$ at $37^{\circ} \mathrm{C}$ exposed to sub-inhibitory concentrations $(100$ or $250 \mu \mathrm{M})$ of CORM-2 ( $\left[\mathrm{Ru}(\mathrm{CO})_{3} \mathrm{Cl}_{2}\right]_{2}$ (Sigma-Aldrich, St. Louis, MO, USA) or left untreated. After an additional $18 \mathrm{~h}$ of incubation under static conditions at $37{ }^{\circ} \mathrm{C}$, biofilm formation was quantified by the crystal violet method as previously described [25]. The absorbance at $540 \mathrm{~nm}$ was measured by spectrophotometer (Thermo Labsystems, Multiscan Ascent).

\section{Live/dead viability staining assay}

Overnight cultures of biofilm producing strains were grown on eight-well chambered cover glasses (Lab-Tek, Rochester NY) for $24 \mathrm{~h}$ at $37{ }^{\circ} \mathrm{C}$. Planktonic bacteria were removed by gentle washing and the remaining biofilm was exposed to CORM-2 $(500 \mu \mathrm{M})$, cefotaxime $(0.512 \mu \mathrm{g} / \mathrm{ml}$ (Sigma-Aldrich), corresponding to $4 \mathrm{x}$ MIC for UPEC isolate 2) or vehicle (DMSO $2.5 \%$ or sterile water) for $24 \mathrm{~h}$ at $37{ }^{\circ} \mathrm{C}$. MIC for CORM-2 has previously been reported to be $500 \mu \mathrm{M}$ in E. coli [21]. Thereafter, the biofilms were washed with $0.85 \% \mathrm{NaCl}$ and incubated with a live/dead viability staining assay (BacLight, Life Technologies, Leiden, The Netherlands) according to kit instructions. Biofilm images were obtained using a Leica TCS confocal laser scanning microscope (Leica Microsystems, Heidelberg GmbH, Wetzlar, Germany). The ratio of dead bacteria (expressed as a percentage) was calculated by manual counting of four randomly selected quadrants (in total covering $1 / 4$ from each image) from a representative part of each well.

\section{Bacterial viability under planktonic growth conditions}

Overnight LB-cultures were used to inoculate (at $0.1 \%$ ) fresh MS-medium. In experiments representing the exponential growth phase, cultures were grown to an $\mathrm{OD}_{620}$ of approximately 0.1 to reach early exponential phase. The bacterial concentration of the initial inoculums used in these experiments was approximately $10^{7}-10^{8} \mathrm{CFU} / \mathrm{ml}$. In experiments representing the stationary growth phase, cultures were grown with aeration at $37{ }^{\circ} \mathrm{C}$ for approximately $14 \mathrm{~h}$. The bacterial concentration of the initial inoculums used in these experiments was approximately $10^{9}$ $\mathrm{CFU} / \mathrm{ml}$. The bacteria were treated with CORM-2 (500 $\mu \mathrm{M})$, DMSO (controls) or cefotaxime $(0.512 \mu \mathrm{g} / \mathrm{ml})$ for 4 and $24 \mathrm{~h}$. After treatment, the bacterial viability was determined by plating serial dilution on TSA plates followed by counting the CFU numbers on overnight cultures at $37{ }^{\circ} \mathrm{C}$. Bacterial $\mathrm{CFU} / \mathrm{ml}$ was determined by using the mean from two dilutions.

\section{Human bladder epithelial cells}

The human bladder epithelial cell line 5637 (ATCC HTB-9; American Type Culture Collection Manassas, USA) was grown in Dulbecco's Modified Eagle medium (Sigma-Aldrich) supplemented with $10 \%$ fetal bovine serum, $2 \mathrm{mM}$ L-glutamine, $1 \mathrm{mM}$ non-essential amino acids, $100 \mathrm{U} / \mathrm{ml}$ penicillin and $100 \mu \mathrm{g} / \mathrm{ml}$ streptomycin (all from Invitrogen, Paisley, UK) in a humidified incubator at $37^{\circ} \mathrm{C}$ with $5 \% \mathrm{CO}_{2}$.

\section{Bacterial viability following colonization of host bladder cells}

Overnight LB-cultures of ESBL-producing isolate 6, 7 and 9 were grown at $37^{\circ} \mathrm{C}$ in static LB-broth to facilitate induction of type-1 fimbriae expression [30]. The 5637 bladder epithelial cells were grown in 24-wells until confluent and infected with bacteria in DMEM supplemented with $2 \%$ fetal bovine serum, $2 \mathrm{mM}$ L-glutamine and $1 \mathrm{mM}$ non-essential amino acids. A multiplicity of infection (MOI) of approximately 20 bacteria per host cells was used. The 24 well plates were centrifuged at $600 \mathrm{x} \mathrm{g}$ for $10 \mathrm{~min}$ to facilitate the attachment of bacteria to epithelial cells followed by incubation for $2 \mathrm{~h}$. A gentamycin protection assay was used to support intracellular growth of UPEC as previously described [12]. 
Briefly, cells were incubated with $100 \mu \mathrm{g} / \mathrm{ml}$ of gentamycin in DMEM for $2 \mathrm{~h}$ to kill extracellular bacteria. Following washes with PBS, the cells were incubated with DMEM containing a submaximal concentration of gentamycin $(10 \mu \mathrm{g} / \mathrm{ml})$ for another $14 \mathrm{~h}$ to limit potential leak of gentamycin into the bladder cells. Cell layers were washed and exposed for $4 \mathrm{~h}$ to CORM-2 (250 and $500 \mu \mathrm{M})$, cefotaxime $(0.512 \mu \mathrm{g} / \mathrm{ml})$ or controls (DMSO or $\mathrm{Ru}(\mathrm{DMSO})_{4} \mathrm{Cl}_{2}$ ). $\mathrm{Ru}(\mathrm{DMSO})_{4} \mathrm{Cl}_{2}$ (Strem chemicals Inc, Newburyport, MA, USA) is a negative control for CORM-2 where the CO groups have been replaced by DMSO. The host cell viability and adherence were checked throughout the experiments. After incubation, the cells were washed, lysed with $0.5 \%$ Triton X-100 and serially diluted and counted by plating on TSA agar plates as described before.

\section{Immunofluorescence of UPEC in the presence of host bladder epithelial cells}

Bladder 5637 cells were seeded onto well glass chamber slides (SPL Lifesciences Co., Ltd., Pocheon-city, GyeonggiDo, Korea), grown to subconfluency and infected with ESBL isolates as described above. After the incubation with gentamycin (totally $16 \mathrm{~h}$ ), the cells were washed with PBS and fixed for $10 \mathrm{~min}$ in $4 \%$ paraformaldehyde. Cells were washed and incubated with $1 \%$ bovine serum albumine (BSA) to block unspecific binding of the antibodies. Extracellular bacteria were labelled by incubation with a goat polyclonal anti-E. coli antibody (Abcam, Cambridge, UK) diluted 1:500 in PBS with $1 \%$ BSA for $60 \mathrm{~min}$ at room temperature (RT), washed and then incubated with a secondary donkey anti-goat IgG-DyLight549 conjugated antibody (Jackson ImmunoResearch Europe Ltd., Suffolk, UK) (red fluorescence) diluted 1:500 (in PBS with $1 \%$ BSA) for 90 min at RT. In order to label intracellular bacteria the host cells were permeabilized with $0.5 \%$ Triton X-100 for $10 \mathrm{~min}$, washed and reprobed with the goat anti-E. coli antibody for $60 \mathrm{~min}$. After wash, the bacteria were stained with a secondary rabbit anti-goat IgG-FITC conjugated antibody (Sigma-Aldrich) (green fluorescence) diluted 1:400 (in PBS with $1 \% \mathrm{BSA}$ ) for $100 \mathrm{~min}$ at RT. Samples were mounted with Vectashield Mounting media (Vector Laboratories Inc., Burlingame, CA, USA) containing 4', 6-diamidino-2-phenylindole (DAPI) for staining of nuclei. Bacteria that stained both red and green were scored as adherent extracellular bacteria, while those that stained only green were scored as intracellular bacteria. Images were obtained and processed using an Olympus BX60 fluorescence microscope equipped with an Olympus DP71 camera and Adobe Photoshop software.

\section{Statistical analysis}

Data are expressed as mean \pm SEM. Differences between two groups were assessed by the unpaired two-tailed
Students $t$-test or by the one sample $t$-test when controls were normalized to 1 . One-way ANOVA followed by Bonferroni test was used for multiple comparisons. Results were considered statistically significant at $p<0.05$. $\mathrm{n}=$ number of independent experiments.

\section{Results}

\section{Effect of CORM-2 on biofilm formation}

A detectable basal biofilm formation $\left(\mathrm{A}_{540} \geq 0.1\right)$ was found in ESBL-producing isolate 1, UPEC isolates 2 and 3 and the non-pathogenic E. coli $\mathrm{K}-12$ strains MG1655 and TG1 (Table 2). Only these isolates were used for further biofilm studies. A sub-inhibitory concentration of CORM-2 $(250 \mu \mathrm{M})$ significantly increased biofilm production in ESBL-producing isolate $1(p=0.017)$ and in UPEC isolate $2(p=0.0058)$ (Fig. 1$)$. A lower concentration of CORM-2 $(100 \mu \mathrm{M})$ had only minor effects on biofilm formation (data not shown). TG1, a well-established biofilm-producing E. coli $\mathrm{K}-12$ strain, showed reduced biofilm formation $(p=0.0045)$ in response to CORM-2. Biofilm formation in UPEC isolates 3 and the K-12 strain MG1655 was not affected by CORM-2 (Fig. 1).

\section{Effect of CORM-2 on bacterial viability within an established biofilm}

We next examined the effect of CORM-2 $(500 \mu \mathrm{M})$ on UPEC isolate 2, ESBL-producing isolate 1 and $\mathrm{K}-12$ strain TG1 when grown within an established biofilm (biofilm formed for $24 \mathrm{~h}$ on plastic surface). To quantify the effects of CORM-2 on bacterial viability a live/dead viability staining assay and confocal microscopy were used. Pilot experiments showed that $4 \mathrm{~h}$ of exposure to CORM-2 had minor effect on viability but that the viability of all isolates was clearly reduced by CORM-2 (500 $\mu \mathrm{M}$ ) after $24 \mathrm{~h}$ of exposure (Fig. 2). The percentage dead bacteria was $60 \pm 16,61 \pm 18$ and $85 \pm 9$ for ESBL isolate 1, UPEC isolate 2 and strain TG1, respectively (Fig. 2, Table 3). Cefotaxime $(0.512 \mu \mathrm{g} / \mathrm{ml})$, a hydrophilic drug with low permeable [31,32], killed UPEC isolate 2 $(76 \pm 8 \%)$ and long filamentous bacteria were observed by confocal microscopy (Fig. 2). However, no effect on viability was noted by cefotaxime in ESBL-producing isolate 1 or the K-12 strain TG1 (Fig. 2, Table 3). The number of dead bacteria in DMSO-treated controls was low (Fig. 2, Table 3). The CO-free molecule $\mathrm{Ru}(\mathrm{DMSO})_{4} \mathrm{Cl}_{2}$, used as a negative control for CORM-2, had similar effects as DMSO on viability (data not shown).

\section{Effect of CORM-2 on planktonic bacteria in different growth phases}

The antibacterial effect of CORM-2 was compared in planktonic cultures (ESBL-producing isolate 1, UPEC isolate 2 and strain TG1) when exposed in the stationary growth phase or in the exponential growth phase. 
Table 2 The basal biofilm production of the different isolates

\begin{tabular}{lll}
\hline$A_{540}<0.1$ & $A_{540} 0.1-1$ & $A_{540}>1$ \\
\hline ESBL 6, ESBL 7, ESBL 9, UPEC 4, UPEC 5 & ESBL 1, UPEC 2, UPEC 3, MG1655 & TG1 \\
\hline
\end{tabular}

Basal biofilm production after $24 \mathrm{~h}$ in ESBL-producing isolates (ESBL), non-ESBL-producing UPEC isolates (UPEC) and non-pathogenic E. coli K-12 strains (MG1655 and TG1) evaluated by the crystal violet method and measured by absorbance levels $\left(A_{540}\right)$. Detectable biofilm production was considered at $A_{540} \geq 0.1$. Mean values from three to six independent experiments

Unexposed controls in the exponential growth phase showed an increased growth of $\sim 1.5 \log$ units during the $24 \mathrm{~h}$ experiment (Fig. 3a). CORM-2 $(500 \mu \mathrm{M})$ evoked a fast bactericidal effect, in all isolates, with a reduction of bacterial counts by $>3 \log$ units within $4 \mathrm{~h}$ (Fig. 3a). Unexposed controls in the stationary growth phase showed no increased growth during the $24 \mathrm{~h}$ experiment (Fig. 3b). CORM-2 demonstrated a delayed inhibitory response in the stationary growth phase, but a bactericidal effect was found after $24 \mathrm{~h}$ (Fig. 3b). Separate experiments showed that colonies of ESBLproducing isolate 1 that survived CORM-2 treatment in the stationary phase, was effectively killed when reexposed in the exponential growth phase. Planktonic cultures of UPEC isolate 2 and the K-12 strain TG1 were susceptible to cefotaxime with a reduction of growth by $0.32 \pm 0.047$ and $1.5 \pm 0.47 \log$ units, respectively. ESBLproducing isolate 1 was resistant to cefotaxime.

\section{Effect of CORM-2 following colonization of host bladder epithelial cells}

Separate experiments demonstrated that ESBL-producing isolates 7 and 9 showed weak colonization of human 5637 bladder epithelial cells, while ESBL isolate 6 showed a

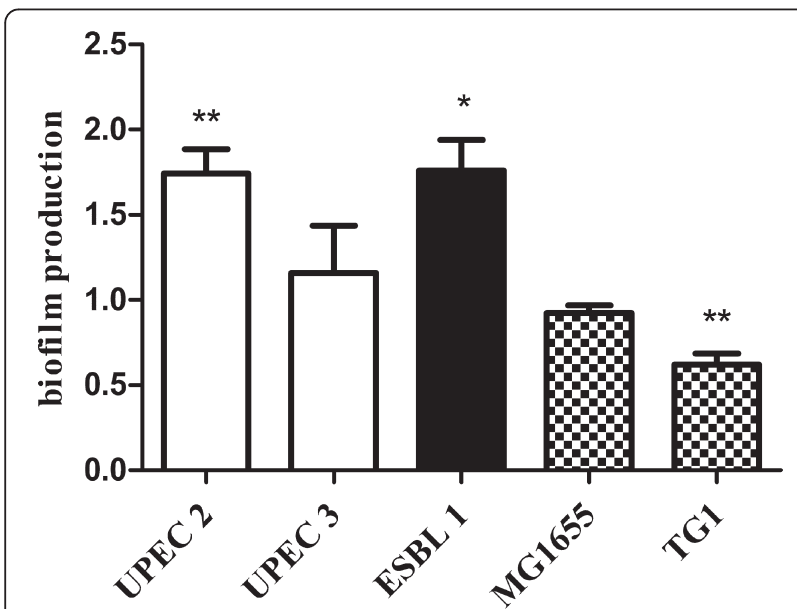

Fig. 1 The antibacterial effect of CORM-2 on biofilm formation. Effect of CORM-2 (250 $\mu \mathrm{M})$ on biofilm formation in non-ESBL-producing UPEC isolates (UPEC 2 and 3), in an ESBL-producing isolate (ESBL1) and in the non-pathogenic E. coli K-12 strains MG1655 and TG1. Biofilm formation was measured by the crystal violet method $18 \mathrm{~h}$ after exposure to CORM-2 and expressed as relative changes compared to untreated controls. The data are presented as mean \pm SEM from at least three independent experiments. ${ }^{*} P<0.05,{ }^{*} P<0.01$, CORM-2 versus control consistent ability to colonize host bladder cells. ESBL isolate 1 was excluded in the colonization experiments due to its gentamycin resistant profile. Infected 5637 bladder cells were less confluent than uninfected 5637 cells and approximately $50 \%$ of the cells were viable after $16 \mathrm{~h}$ of infection. Exposure to CORM-2 at $500 \mu \mathrm{M}$, but not 250 $\mu \mathrm{M}$, for $4 \mathrm{~h}$ significantly $(p=0.0235)$ reduced the bacterial counts of ESBL isolate 6 in the presence of 5637 bladder cells by approximately $2.5 \log$ units (Fig. 4a). A negative control for CORM-2, $\mathrm{Ru}(\mathrm{DMSO})_{4} \mathrm{Cl}_{2}$, and cefotaxime did not reduce the bladder cell colonization of ESBL isolate 6 (Fig. 4a). CORM-2 did not affect 5637 bladder cell confluence or adherence during the $4 \mathrm{~h}$ exposure time. A double immunofluorescence staining procedure was performed to investigate the localization of colonized bacteria. Bacteria were found both attached to host bladder cells in the extracellular space and also intracellular (Fig. 4b-d).

\section{Discussion}

Several recent publications highlight the urgent need for new therapies against multidrug-resistant $E$. coli $[33,34]$. Multidrug-resistant UPEC associated with biofilm on urinary catheters and within bladder reservoirs are particularly problematic to eradicate due to a combination of antibiotic resistance and antibiotic penetration barriers. In the present study, the overall biofilm formation on plastic abiotic surface was low in the UPEC isolates compared to the positive control E. coli $\mathrm{K}-12$ strain TG1. A significant variation in the capacity of different E. coli isolates to form biofilm exists and both genetic and environmental factors affect the biofilm phenotype in a complex manner [35]. Growth medium composition has significant impact on biofilm formation [35] and some virulence-associated genes appear to be more common in E. coli strains with strong biofilm production [36]. A flow chamber biofilm model system would possibly have improved the biofilm formation of the UPEC isolates in the present study. When UPEC isolates with detectable biofilm formation were exposed to CORM-2 an increased biofilm formation was found in two out of three isolates, suggesting that increased biofilm production may be a defence mechanism against CORM-2. A previous study performed in an E. coli $\mathrm{K}-12$ strain showed that CORM-2 increased transcription of several biofilm-related genes and increased biofilm production when grown in LB-broth [25]. The two non-pathogenic 


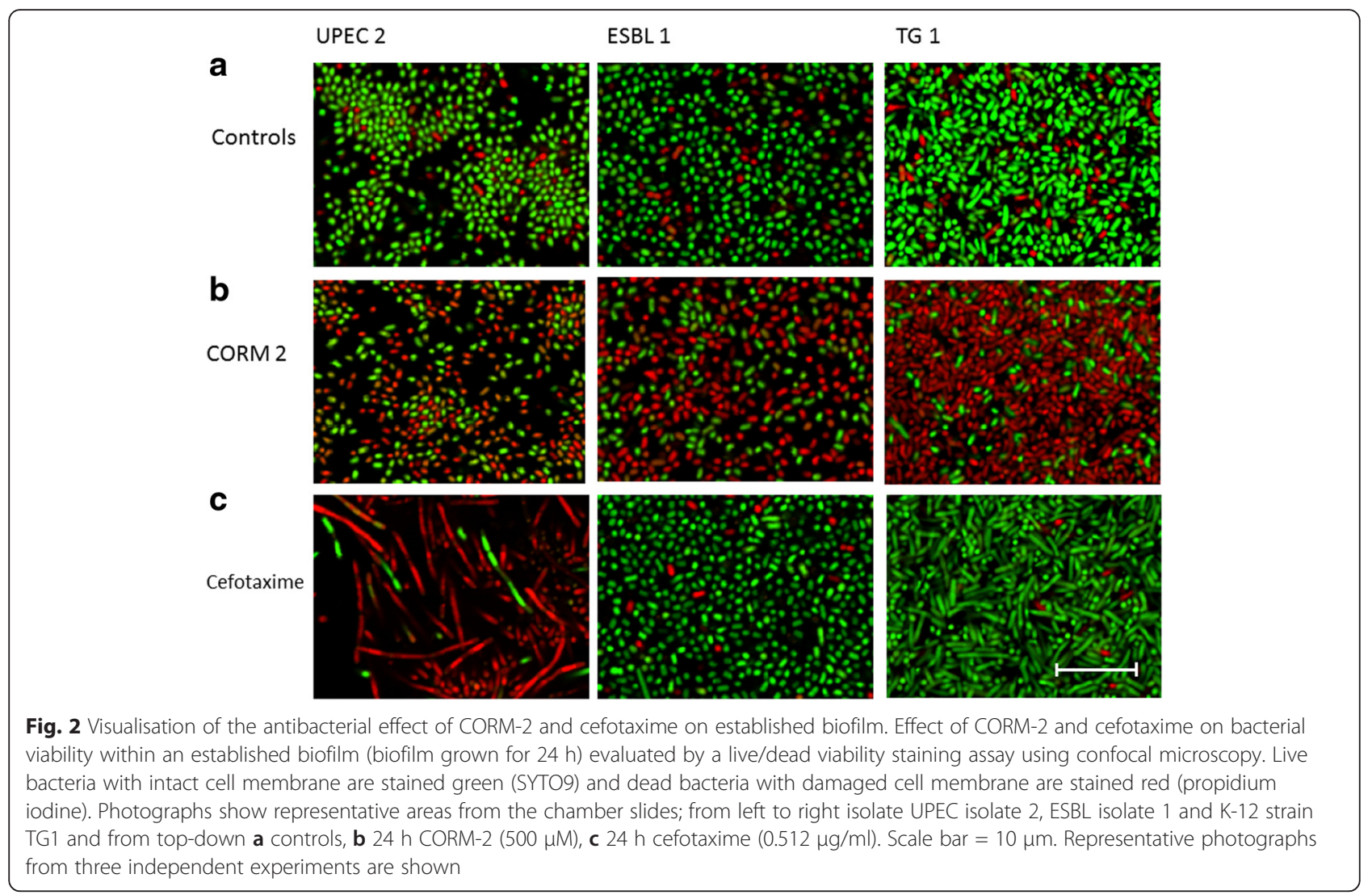

K-12 strains used in our study were unaffected or showed a reduced biofilm formation when exposed to CORM-2. Biofilm formation was examined in a concentration $(250 \mu \mathrm{M})$ that is sub-inhibitory for UPEC [26], however the growth of planktonic TG1 was reduced at this concentration (data not shown), which may explain the impaired ability to form biofilm.

Table 3 Quantitative data from the live/dead viability assay

\begin{tabular}{lcc}
\hline Stimuli & Mean $\% \pm$ SEM & $P$ value \\
\hline UPEC 2 control & $5 \pm 2$ & \\
UPEC 2 CORM-2 & $61 \pm 18$ & $0.0208^{*}$ \\
UPEC 2 cefotaxime & $76 \pm 8$ & $0.0011^{* *}$ \\
ESBL 1 control & $6 \pm 2$ & \\
ESBL 1 CORM-2 & $60 \pm 16$ & $0.0135^{*}$ \\
ESBL 1 cefotaxime & $5 \pm 1$ & \\
TG1 control & $13 \pm 2$ & $0.0003^{* * *}$ \\
TG1 CORM-2 & $85 \pm 9$ & \\
TG1 cefotaxime & $4 \pm 1$ &
\end{tabular}

Data show the ratio of dead bacteria (expressed as a percentage) after $24 \mathrm{~h}$ exposure to CORM-2 $(500 \mu \mathrm{M})$, cefotaxime $(0.512 \mu \mathrm{g} / \mathrm{ml})$ or control (DMSO) evaluated by a live/dead viability assay. The ratio of dead bacteria was calculated by manual counting of four randomly selected quadrants (in total covering $1 / 4$ from each image) from a representative part of each well. The data are presented as mean \pm SEM. $(n=3){ }^{*} P<0.05,{ }^{* *} P<0.01,{ }^{* * *} P<0.001$; CORM-2 or cefotaxime versus control
CORMs represent a novel class of antimicrobials and are so far non-explored agents in the field of eradication of biofilm growth caused by UPEC. The CO-releasing profile of CORMs has recently been re-evaluated [20]. It has been proposed that CORMs are internalized into bacteria by a Trojan horse mechanism, rather than that CO diffuses into the cell $[22,37]$. CORM-2 was able to reduce the bacterial viability of ESBL-producing and non-ESBL-producing UPEC isolates, as well as the K12 strain TG1, when grown within an established biofilm. Thus, CORM-2 seems able to penetrate biofilm-like architecture and kill bacteria in biofilms as confirmed by the live/dead viability assay and confocal microscopy.

The ESBL-producing isolate was resistant to the cephalosporine antibiotic cefotaxime and, as expected, cefotaxime did not affect the viability of the ESBL isolate within an established biofilm. However, exposure to cefotaxime reduced the viability of the non-ESBL producing UPEC isolate and long filamentous bacteria were visualized by confocal microscopy. Filamentous bacteria can appear as a consequence of bacterial stress, including exposure to beta-lactam antibiotics that through inhibition of penicillin-binding protein-3 in the peptidoglycan layer prevents septa formation and cell division [38]. E. coli strain TG1, with the highest biofilm production, was effectively killed ( $85 \%$ ) by CORM-2 but was 


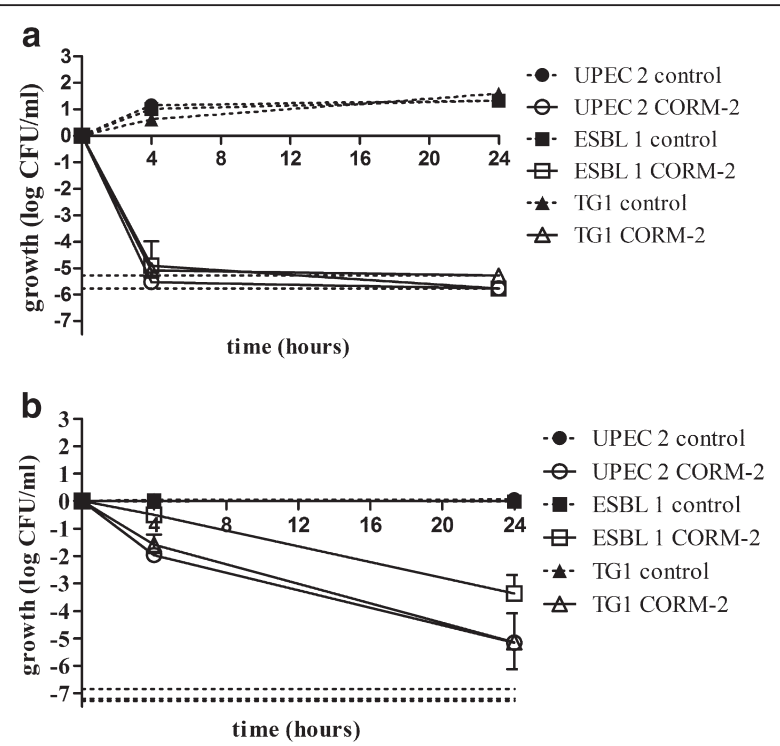

Fig. 3 The antibacterial effect of CORM-2 in the exponential and stationary growth phase. A comparison of the effect of CORM-2 exposure in the exponential growth phase and in the stationary phase in ESBL isolate 1, UPEC isolate 2 and K-12 strain TG1. a Cultures were grown to early log phase in MS-broth and then exposed to CORM-2 (500 $\mu \mathrm{M})$ or DMSO (control). b Cultures were grown to stationary phase in MS-broth and then exposed to CORM$2(500 \mu \mathrm{M})$ or DMSO (control). Growth was calculated as the numbers of $\mathrm{CFU} / \mathrm{ml}$ in treated cultures or controls divided by the number of CFU/ml formed upon the plating of the initial inoculums and expressed as log CFU/ml. The data are presented as mean \pm SEM from at least three independent experiments. Lower grids show the detection limit

insensitive to cefotaxime. Cefotaxime is mainly active against dividing bacteria and has low efficiency for adherent bacteria within a biofilm matrix compared to planktonic cultures [39]. Our data confirmed that planktonic TG1 cultures, but not bacterial biofilms, were sensitive to cefotaxime. A limitation of this study is that the biofilm formation by the clinical UPEC isolates was low, and that the effects of CORM-2 on biofilms may have been overestimated. However, CORM-2 was effective against TG-1 biofilms suggesting that CORM-2 is able to penetrate and reach targets within more mature biofilms. In agreement with our findings, CORM-2 was demonstrated to kill a majority of clinical isolates of $P$. aeruginosa within an established biofilm [40]. The main mechanism by which CORM-2 inhibited $P$. aeruginosa biofilm appeared to be interference with the respiratory chain rather than production of reactive oxygen species [40].

Impaired penetration of antibiotics is not the only mechanism associated with biofilm-related antibiotic resistance. Another characteristic of a biofilm is the presence of a large subpopulation of bacteria in a dormant persister state [38]. Bacteria in a dormant state are found in biofilms and in stationary phase cultures, but not in cultures in the exponential growth phase. Bacteria that have entered a dormant state are transiently tolerant to antibiotics by mechanism such as reduced translation and cell wall synthesis [41, 42]. The effects of CORM-2 in the exponential and stationary growth phase were investigated to reveal if slower growing bacteria are more resistant to CORM-2. CORM-2 reduced bacterial viability in the stationary phase, but the inhibition was markedly delayed compared to cultures in the exponential phase demonstrating that metabolically active bacteria are more susceptible to CORM-2. Bacteria that survived the CORM-2 treatment in stationary phase experiments were killed when re-exposed to CORM-2 in the exponential phase, which suggest that stationary phase bacteria may represent dormant persisters with a transient tolerance to CORM-2. The higher cell density in the stationary phase may in part explain the delayed efficacy of CORM-2 in the stationary phase since the effective drug concentration for each bacterium may be lower.

The 5637 human bladder epithelial cell line was infected with an ESBL-producing isolate and the gentamycin protection assay was used to support intracellular localization. There are known difficulties in replicating the conditions required for formation of intracellular biofilm-like bacterial communities in vitro. However, UPEC have been shown to invade 5637 bladder epithelial cells but they rarely form large IBC inclusions as found in superficial bladder facet cells in vivo [11, 43]. The immunofluorescence method used in the present study, which is able to distinguish between extra- and intracellular bacteria, demonstrated that the ESBL isolate could adhere to and invade 5637 bladder epithelial cells. In agreement with previous studies [11, 43, 44], the intracellular bacteria were dispersed in the cytosol and not packed in biofilm-like aggregates. Exposure to CORM-2 significantly reduced the bacterial counts following bladder epithelial cell colonization. The CO-free molecule $\mathrm{Ru}(\mathrm{DMSO})_{4} \mathrm{Cl}_{2}$ elicited no reduced viability, verifying that $\mathrm{CO}$ is required for the antibacterial effect of CORM-2. The cell cytotoxicity of CORM-2 (100-500 $\mu \mathrm{M})$ has previously been observed to be low in 5637 bladder cells and CORM-2 even seemed to have a cytoprotective effect after $4 \mathrm{~h}$ [26]. The exposure time to CORM-2 was therefore restricted to $4 \mathrm{~h}$ in our experiments and no detrimental effects on the cells were observed. It may be argued that cytotoxic effects of CORM-2 on host bladder cells may enhance its efficacy against internalized UPEC by disrupting cell barriers. However, no correlation between 5637 bladder cell cytotoxicity and killing of intracellular UPEC by several antibiotics was found using a similar cell-culture based assay as in our study [12]. Nevertheless, we cannot exclude that some effects of CORM-2 on the host bladder cells or on bacterial efflux mechanisms from the 5637 cells 

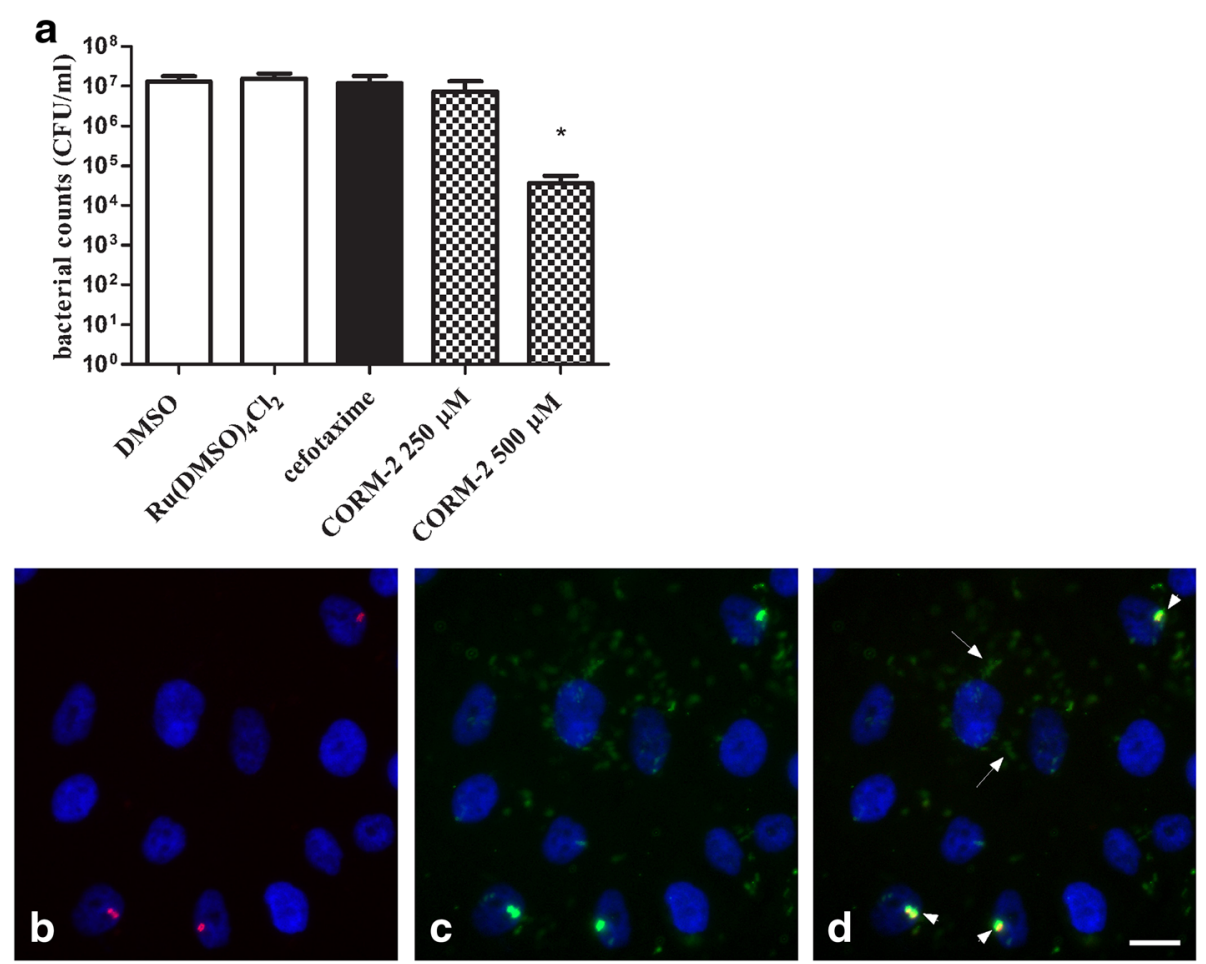

Fig. 4 The antibacterial effect of CORM-2 on colonization of host cells and localization of colonized bacteria. Quantification and immunofluorescence staining of ESBL-producing isolate 6 following colonization of 5637 bladder epithelial cells. A gentamycin protection assay was used to support intracellular growth of the bacteria. a Host bladder 5637 cells were lysed and the lysate serially diluted and plated on TSA plates for quantification of bacterial count (CFU/ml). Bacterial counts in cell lysate was evaluated after exposure for $4 \mathrm{~h}$ to DMSO, the CO-free molecule $\mathrm{Ru}(\mathrm{DMSO})_{4} \mathrm{Cl}{ }_{2}(500 \mu \mathrm{MM})$, cefotaxime $(0.512 \mu \mathrm{g} / \mathrm{ml})$ or CORM-2 $(250$ and $500 \mu \mathrm{M})$. Data are presented as mean \pm SEM from four independent experiments. ${ }^{*} P<0.05$, CORM-2 versus DMSO. b-d Immunofluorescence staining of ESBL-producing isolate 6 following infection of 5637 bladder epithelial cells. Staining of the bladder cell nuclei was performed with DAPI and is shown in blue. ESBL isolate 6 are stained $\mathbf{b}$ ) in red (extracellular) prior to permeabilization, and $\mathbf{c}$ ) in green (extracellular and intracellular) after permeabilization. $\mathbf{d}$ Merged image of B and C where several intracellular bacteria are seen as green stain (arrows) and extracellular bacteria are shown as merged red and green (yellow) stain (arrowhead). Scale bar $=10 \mu \mathrm{m}$

contribute to the antibacterial effects of CORM-2 on colonized bacteria. Taken together, CORM-2 has an interesting antibacterial profile by being effective against multidrug-resistant UPEC protected in biofilms and in intracellular reservoirs. It will be of interest to examine whether CORMs alone or in combination with currently available antibiotics will have antibacterial effect on intracellular and persistent UPEC in mouse UTI models. The search of CORMs with suitable properties for the delivery of $\mathrm{CO}$ and with low cytotoxicity is an ongoing and active area of research. New CORMs with desirable therapeutic profiles and clinical compatibility are appearing, but more fundamental knowledge on the chemistry, cellular targets and molecular biology of CORMs is needed before these compounds can be used clinically as antimicrobial agents $[20,45,46]$.

The ESBL-producing isolates 6 and 7 belong to the $E$. coli ST131 clone that currently represents one of the most dominant groups of multidrug-resistant E. coli globally [47]. The ST131 clone, which produces CTX-15, is thought to be successful due to a combination of antibiotic resistance and virulence. Here, we show that ESBL isolate 6 was able to adhere to and invade host bladder epithelial cells, suggesting that this isolate has a capacity to form intracellular reservoirs and persist in the bladder. In agreement, a ST131 reference strain E. coli EC958 has previously been demonstrated to invade bladder epithelial cells both in mouse studies [48] and in vitro studies [49].

\section{Conclusions}

This study demonstrates that CORM-2 is able to reduce the bacterial viability of multidrug-resistant UPEC in biofilm-like conditions and following colonization of human bladder epithelial cells. CORMs will be interesting candidate drugs to investigate further in studies of recurrent and chronic UTI caused by multidrug-resistant UPEC.

Ethics approval and consent to participate Not applicable. 


\section{Consent for publication Not applicable.}

\section{Availability of data and materials All the data supporting the findings is contained within the manuscript or will be shared upon request.}

\author{
Abbreviations \\ BSA: Bovine serum albumin; CAZ: Ceftazidime; CIP: Ciprofloxacin; CO: Carbon \\ monoxide; CORM-2: Carbon monoxide-releasing molecule-2; CORM- \\ 3: Carbon monoxide-releasing molecule-3; CTB: Ceftibuten; CTX: Cefotaxime; \\ DAPI: 4', 6-diamidino-2-phenylindole; E. coli: Escherichia coli; ESBL: Extended- \\ spectrum $\beta$-lactamase; GEN: Gentamicin; IBC: Intracellular bacterial \\ communities; LB: Luria-Bertani broth; MEL: Mecillinam; MOl: Multiplicity of \\ infection; MS: Minimal salt medium; NIT: Nitrofurantoin; $P$. \\ aeruginosa: Pseudomonas aeruginosa; TMP: Trimethoprim; \\ UPEC: Uropathogenic E. coli; UTI: Urinary tract infection; RT: Room \\ temperature.
}

\section{Competing interests}

The authors declared that they have no competing interests.

\section{Authors' contributions}

Conception and design of the study: KP, CSB. Laboratory work: CSB, RK, KJ. Data analysis and interpretation: CSB, RK, KJ, KP. Manuscript writing: CSB, KP. All authors have read and approved the final manuscript.

\section{Acknowledgements}

We thank Dr Robert Poole (University of Sheffield, U. K) for the gift of bacterial strain TG1 and Anna Önnberg for CTX-M typing and ST131 clone detection of the ESBL-producing isolates.

\section{Funding}

Funding for salary and materials was provided by the Swedish Council for Working Life and Social Research (FAS), the Faculty of Medicine and Health at Örebro University and Nyckelfonden at Örebro University Hospital.

\section{Received: 19 May 2015 Accepted: 29 March 2016}

\section{Published online: 12 April 2016}

\section{References}

1. Costerton JW, Stewart PS, Greenberg EP. Bacterial biofilms: a common cause of persistent infections. Science. 1999;284(5418):1318-22.

2. Van Houdt R, Michiels CW. Role of bacterial cell surface structures in Escherichia coli biofilm formation. Res Microbiol. 2005:156(5-6):626-33.

3. Mah TF, O'Toole GA. Mechanisms of biofilm resistance to antimicrobial agents. Trends Microbiol. 2001;9(1):34-9.

4. Hatt JK, Rather PN. Role of bacterial biofilms in urinary tract infections. Curr Top Microbiol Immunol. 2008;322:163-92.

5. Niveditha S, Pramodhini S, Umadevi S, Kumar S, Stephen S. The Isolation and the Biofilm Formation of Uropathogens in the Patients with Catheter Associated Urinary Tract Infections (UTIs). J Clin Diagn Res. 2012;6(9):1478-82.

6. Soto SM, Smithson A, Horcajada JP, Martinez JA, Mensa JP, Vila J. Implication of biofilm formation in the persistence of urinary tract infection caused by uropathogenic Escherichia coli. Clin Microbiol Infect. 2006;12(10):1034-6.

7. Anderson GG, Palermo JJ, Schilling JD, Roth R, Heuser J, Hultgren SJ. Intracellular bacterial biofilm-like pods in urinary tract infections. Science. 2003:301(5629):105-7.

8. Dhakal BK, Kulesus RR, Mulvey MA. Mechanisms and consequences of bladder cell invasion by uropathogenic Escherichia coli. Eur J Clin Invest. 2008;38 Suppl 2:2-11.

9. Scott VC, Haake DA, Churchill BM, Justice SS, Kim JH. Intracellular Bacterial Communities: A Potential Etiology for Chronic Lower Urinary Tract Symptoms. Urology. 2015;86(3):425-31.

10. Mysorekar IU, Hultgren SJ. Mechanisms of uropathogenic Escherichia coli persistence and eradication from the urinary tract. Proc Natl Acad Sci U S A. 2006:103(38):14170-5.

11. Eto DS, Sundsbak JL, Mulvey MA. Actin-gated intracellular growth and resurgence of uropathogenic Escherichia coli. Cell Microbiol. 2006;8(4): 704-17.
12. Blango MG, Mulvey MA. Persistence of uropathogenic Escherichia coli in the face of multiple antibiotics. Antimicrob Agents Chemother. 2010; 54(5):1855-63.

13. Schilling JD, Lorenz RG, Hultgren SJ. Effect of trimethoprim-sulfamethoxazole on recurrent bacteriuria and bacterial persistence in mice infected with uropathogenic Escherichia coli. Infect Immun. 2002;70(12):7042-9.

14. Oteo J, Perez-Vazquez M, Campos J. Extended-spectrum [beta]-lactamase producing Escherichia coli: changing epidemiology and clinical impact. Curr Opin Infect Dis. 2010;23(4):320-6.

15. Hoban DJ, Lascols C, Nicolle LE, Badal R, Bouchillon S, Hackel M, Hawser S. Antimicrobial susceptibility of Enterobacteriaceae, including molecular characterization of extended-spectrum beta-lactamase-producing species, in urinary tract isolates from hospitalized patients in North America and Europe: results from the SMART study 2009-2010. Diagn Microbiol Infect Dis. 2012;74(1):62-7.

16. Pitout JD. Extraintestinal Pathogenic Escherichia coli: A Combination of Virulence with Antibiotic Resistance. Front Microbiol. 2012;3:9.

17. Peirano G, Pitout JD. Molecular epidemiology of Escherichia coli producing CTX-M beta-lactamases: the worldwide emergence of clone ST131 O25:H4 Int J Antimicrob Agents. 2010;35(4):316-21.

18. Fernandez $\mathrm{L}$, Breidenstein EB, Hancock RE. Creeping baselines and adaptive resistance to antibiotics. Drug Resist Updat. 2011;14(1):1-21.

19. Li L, Moore PK. An overview of the biological significance of endogenous gases: new roles for old molecules. Biochem Soc Trans. 2007;35(Pt 5):1138-41.

20. Garcia-Gallego S, Bernardes GJ. Carbon-Monoxide-Releasing Molecules for the Delivery of Therapeutic CO In Vivo. Angew Chem (International ed in English). 2014;53(37):9712-21.

21. Nobre LS, Seixas JD, Romao CC, Saraiva LM. Antimicrobial action of carbon monoxide-releasing compounds. Antimicrob Agents Chemother. 2007; 51(12):4303-7.

22. Davidge KS, Sanguinetti G, Yee CH, Cox AG, McLeod CW, Monk CE, Mann BE, Motterlini R, Poole RK. Carbon monoxide-releasing antibacterial molecules target respiration and global transcriptional regulators. J Biol Chem. 2009;284(7):4516-24.

23. Desmard M, Davidge KS, Bouvet O, Morin D, Roux D, Foresti R, Ricard JD, Denamur E, Poole RK, Montravers P, et al. A carbon monoxide-releasing molecule (CORM-3) exerts bactericidal activity against Pseudomonas aeruginosa and improves survival in an animal model of bacteraemia. FASEB J. 2009:23(4):1023-31.

24. Tavares AF, Teixeira M, Romao CC, Seixas JD, Nobre LS, Saraiva LM. Reactive oxygen species mediate bactericidal killing elicited by carbon monoxidereleasing molecules. J Biol Chem. 2011;286(30):26708-17.

25. Nobre LS, Al-Shahrour F, Dopazo J, Saraiva LM. Exploring the antimicrobial action of a carbon monoxide-releasing compound through whole-genome transcription profiling of Escherichia coli. Microbiology. 2009;155(Pt 3):813-24.

26. Bang CS, Kruse R, Demirel I, Onnberg A, Soderquist B, Persson K. Multiresistant uropathogenic extended-spectrum beta-lactamase (ESBL)producing Escherichia coli are susceptible to the carbon monoxide releasing molecule-2 (CORM-2). Microb Pathog. 2014;66:29-35.

27. Onnberg A, Molling P, Zimmermann J, Soderquist B. Molecular and phenotypic characterization of Escherichia coli and Klebsiella pneumoniae producing extended-spectrum beta-lactamases with focus on CTX-M in a low-endemic area in Sweden. APMIS. 2011;119(4-5):287-95.

28. Dhanji H, Doumith M, Clermont O, Denamur E, Hope R, Livermore DM, Woodford N. Real-time PCR for detection of the O25b-ST131 clone of Escherichia coli and its CTX-M-15-like extended-spectrum beta-lactamases. Int J Antimicrob Agents. 2010;36(4):355-8.

29. Latimer J, Stokes SL, Graham Al, Bunch J, Jackson RJ, McLeod CW, Poole RK A novel method for exploring elemental composition of microbial communities: laser ablation-inductively coupled plasma-mass spectrometry of intact bacterial colonies. J Microbiol Methods. 2009;79(3):329-35.

30. Martinez JJ, Mulvey MA, Schilling JD, Pinkner JS, Hultgren SJ. Type 1 pilus-mediated bacterial invasion of bladder epithelial cells. EMBO J. 2000;19(12):2803-12.

31. Amidon GL, Lennernas H, Shah VP, Crison JR. A theoretical basis for a biopharmaceutic drug classification: the correlation of in vitro drug product dissolution and in vivo bioavailability. Pharm Res. 1995;12(3):413-20.

32. Varma MV, Khandavilli S, Ashokraj Y, Jain A, Dhanikula A, Sood A, Thomas NS, Pillai O, Sharma P, Gandhi R, et al. Biopharmaceutic classification system: a scientific framework for pharmacokinetic optimization in drug research. Curr Drug Metab. 2004;5(5):375-88. 
33. Foxman B. The epidemiology of urinary tract infection. Nat Rev Urol. 2010; 7(12):653-60.

34. Livermore DM. Fourteen years in resistance. Int J Antimicrob Agents. 2012; 39(4):283-94.

35. Reisner A, Krogfelt KA, Klein BM, Zechner EL, Molin S. In vitro biofilm formation of commensal and pathogenic Escherichia coli strains: impact of environmental and genetic factors. J Bacteriol. 2006;188(10):3572-81.

36. Naves P, del Prado G, Huelves L, Gracia M, Ruiz V, Blanco J, Dahbi G, Blanco M, Ponte Mdel C, Soriano F. Correlation between virulence factors and in vitro biofilm formation by Escherichia coli strains. Microb Pathog. 2008;45(2): 86-91.

37. McLean S, Mann BE, Poole RK. Sulfite species enhance carbon monoxide release from CO-releasing molecules: implications for the deoxymyoglobin assay of activity. Anal Biochem. 2012;427(1):36-40.

38. Gould IM, MacKenzie FM. The response of Enterobacteriaceae to betalactam antibiotics-'round forms, filaments and the root of all evil'. J Antimicrob Chemother. 1997:40(4):495-9.

39. Dracea O, lordache C, Bucur M, Bleotu C, Banu O, Ungureanu C, Cristea D, Lixandru MS, Larion C, Necula G, et al. Investigation of the influence of different physico-chemical parameters upon the susceptibility of planktonic and adherent Escherichia coli cells to beta-lactams and quinolones. Roum Arch Microbiol Immunol. 2009:68(1):50-4.

40. Murray TS, Okegbe C, Gao Y, Kazmierczak BI, Motterlini R, Dietrich LE, Bruscia EM. The carbon monoxide releasing molecule CORM-2 attenuates Pseudomonas aeruginosa biofilm formation. PLoS One. 2012;7(4), e35499.

41. Lewis K. Persister cells. Annu Rev Microbiol. 2010;64:357-72.

42. Lewis K. Persister cells, dormancy and infectious disease. Nat Rev Microbiol. 2007;5(1):48-56.

43. Mulvey MA, Schilling JD, Hultgren SJ. Establishment of a persistent Escherichia coli reservoir during the acute phase of a bladder infection. Infect Immun. 2001;69(7):4572-9.

44. Berry RE, Klumpp DJ, Schaeffer AJ. Urothelial cultures support intracellular bacterial community formation by uropathogenic Escherichia coli. Infect Immun. 2009;77(7):2762-72.

45. McLean S, Begg R, Jesse HE, Mann BE, Sanguinetti G, Poole RK. Analysis of the Bacterial Response to $\mathrm{Ru}(\mathrm{CO}) \mathrm{Cl}(\mathrm{Glycinate})(\mathrm{CORM}-3)$ and the Inactivated Compound Identifies the Role Played by the Ruthenium Compound and Reveals Sulfur-Containing Species as a Major Target of CORM-3 Action. Antioxid Redox Signal. 2013.

46. Wareham LK, Poole RK, Tinajero-Trejo M. CO-releasing Metal Carbonyl Compounds as Antimicrobial Agents in the Post-antibiotic Era. J Biol Chem. 2015;290(31):18999-9007.

47. Woodford N, Turton JF, Livermore DM. Multiresistant Gram-negative bacteria: the role of high-risk clones in the dissemination of antibiotic resistance. FEMS Microbiol Rev. 2011;35(5):736-55.

48. Totsika M, Kostakioti M, Hannan TJ, Upton M, Beatson SA, Janetka JW, Hultgren SJ, Schembri MA. A FimH inhibitor prevents acute bladder infection and treats chronic cystitis caused by multidrug-resistant uropathogenic Escherichia coli ST131. J Infect Dis. 2013;208(6):921-8.

49. Totsika M, Beatson SA, Sarkar S, Phan MD, Petty NK, Bachmann N, Szubert M, Sidjabat HE, Paterson DL, Upton M, et al. Insights into a multidrug resistant Escherichia coli pathogen of the globally disseminated ST131 lineage: genome analysis and virulence mechanisms. PLoS One. 2011;6(10), e26578.

\section{Submit your next manuscript to BioMed Central and we will help you at every step:}

- We accept pre-submission inquiries

- Our selector tool helps you to find the most relevant journal

- We provide round the clock customer support

- Convenient online submission

- Thorough peer review

- Inclusion in PubMed and all major indexing services

- Maximum visibility for your research

Submit your manuscript at www.biomedcentral.com/submit
Biomed Central 